\title{
Aneurisma de veia poplítea: relato de caso e revisão de literatura
}

\section{Popliteal vein aneurysm: case report and review of the literature}

\author{
Fernando Thomazinho, Jose Antonio Morselli Diniz, Ramzi Abdallah EI Hosni Junior, \\ Carlos Alberto Morselli Diniz, Igor Schincariol Perozin*
}

\begin{abstract}
Resumo
Os aneurismas venosos são entidades raras, porém com potencialidade de causar complicações tromboembólicas. Na maioria das vezes, são encontrados incidentalmente, como achados de exame físico ou de imagem. Os aneurismas sintomáticos de veia poplítea são obrigatoriamente tratados por reparo cirúrgico, devido ao alto risco de recorrência de embolia pulmonar. A técnica mais utilizada é a aneurismectomia tangencial com venorrafia lateral. $\mathrm{Na}$ impossibilidade de se empregar essa técnica, faz-se a ressecção com reconstrução venosa. Os autores relatam o caso de uma paciente com aneurisma de veia poplítea, cujo diâmetro era de $47 \mathrm{~mm}$, submetido a aneurismectomia tangencial e venorrafia lateral, com sucesso.
\end{abstract}

Palavras-chave: Aneurisma, veia, cirurgia.

\section{Introdução}

O termo aneurisma é utilizado para caracterizar uma dilatação focal dos vasos sangüíneos. É freqüentemente relacionado às dilatações das artérias, entretanto essas lesões podem ocorrer em qualquer parte do sistema vascular $^{1}$.

Aneurismas venosos são pouco freqüentes e geralmente aparecem como achados incidentais no exame físico ou nos exames de imagens. Raramente têm significância clínica, com exceção dos aneurismas de veia poplítea (AVP), que devem ser lembrados como uma rara causa de embolia pulmonar recorrente e de trombose venosa profunda ${ }^{2-6}$. A maioria dos aneurismas venosos é de origem congênita, embora também possam ser adquiridos por trauma, processos inflamatórios e alterações degenerativas ${ }^{7}$.

\begin{abstract}
Venous aneurysms are considered to be a rare disease; however they can be a potential cause for the development of thromboembolism. They are mostly detected by physical examination or imaging exams. Symptomatic aneurysms of the popliteal vein must be surgically treated, due to high risk of recurrent pulmonary embolism. The most widely used procedure is tangential aneurysmectomy and lateral venorrhaphy. If not possible, the aneurysm should be removed and a venous reconstruction should be performed. The authors report a case of a patient with a popliteal vein aneurysm measuring $47 \mathrm{~mm}$ in diameter. Tangential aneurysmectomy and lateral venorrhaphy were successfully used for treatment.
\end{abstract}

Keywords: Aneurysm, vein, surgery.

\section{Relato de caso}

Trata-se de paciente de sexo feminino, de cor branca, aposentada, com 60 anos de idade, queixando-se de dor semelhante a cansaço na face póstero-medial da perna esquerda, predominantemente na fossa poplítea. Foi submetida a safenectomia bilateral há 30 anos, apresentando úlcera cicatrizada no tornozelo esquerdo. É portadora de hipotiroidismo controlado e não possui antecedentes de trombose venosa profunda ou dispnéia.

Ao exame físico, constatou-se a presença de varizes tronculares recidivadas com leve dermatofibrose e dermatite ocre na perna esquerda. Apresentava também pequeno abaulamento na fossa poplítea esquerda quando em posição ortostática, que desaparecia com a elevação do membro.

* Hospital Evangélico de Londrina, Londrina, PR.

Não foram declarados conflitos de interesse associados à publicação deste artigo.

Artigo submetido em 25.10.07, aceito em 01.08.08. 


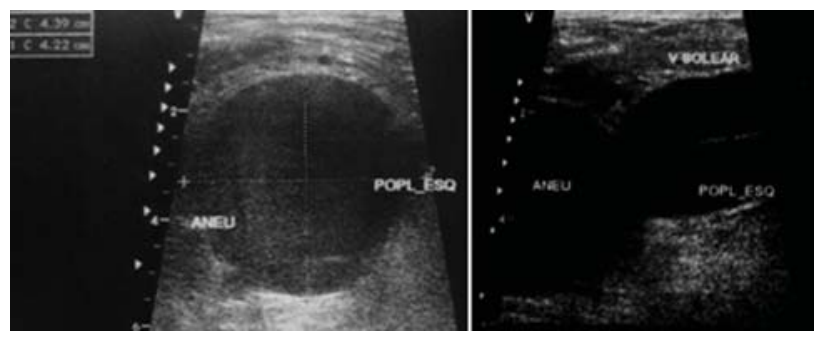

Figura 1 - Ultra-sonografia vascular evidenciando aneurisma da veia poplítea

O exame eco-Doppler evidenciou refluxo no sistema venoso profundo no membro inferior esquerdo, dilatação fusiforme da veia poplítea (Figura 1), com diâmetros transversos de $47 \times 37 \mathrm{~mm}$, e ectasia da veia solear e tibial posterior proximal. Realizou-se também flebografia ascendente, que confirmou os achados (Figura 2).

A paciente foi submetida a aneurismectomia tangencial com venorrafia lateral (Figura 3), por abordagem poplítea posterior. Recebeu alta com prescrição de anticoagulantes, por via oral, durante 90 dias.

O exame de controle através de eco-Doppler seriado demonstrou sistema profundo pérvio com calibre da veia poplítea de $12 \times 13 \mathrm{~mm}$ ao longo de seguimento de 9 meses. Houve melhora dos sintomas relatados previamente.

\section{Discussão}

A primeira publicação sobre aneurisma venoso foi feita por Osler em 1915, que relatou um caso de dilatação da veia axilar ${ }^{8}$. Posteriormente, em 1928, Harris ${ }^{1,3}$

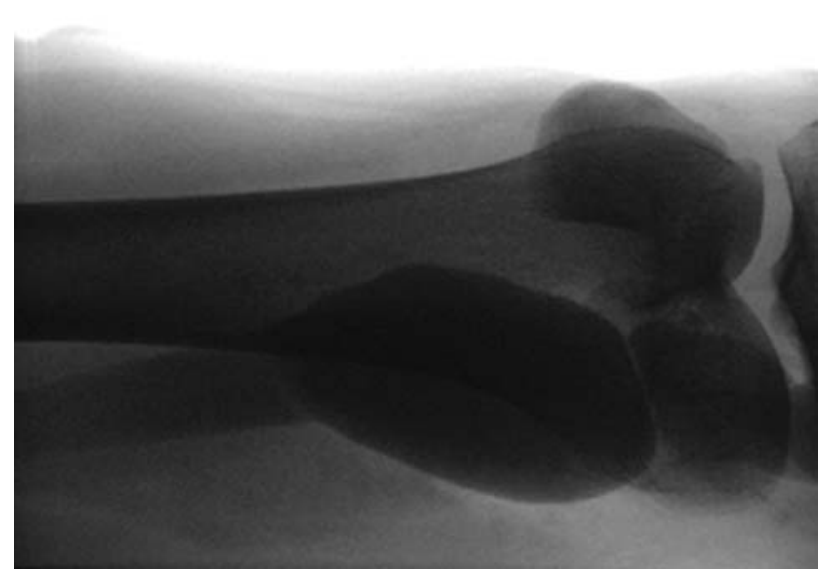

Figura 2 - Flebografia ascendente evidenciando aneurisma fusiforme da veia poplítea

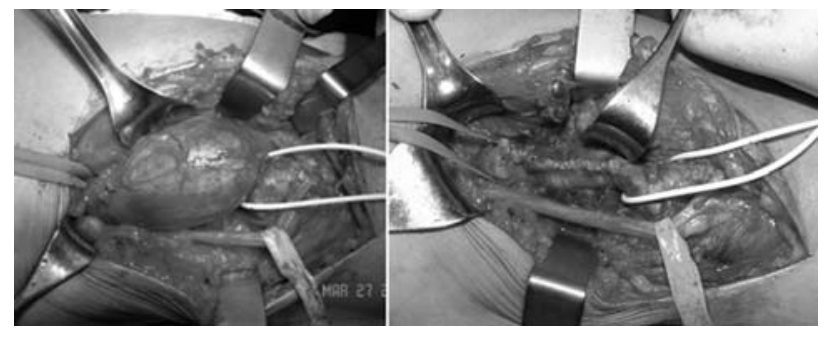

Figura 3 - Aspecto intra-operatório do aneurisma de veia poplítea e resultado após aneurismectomia com venorrafia lateral

descreveu uma criança de 5 anos com aneurisma de veia jugular. Abbott ${ }^{3}$ descreveu um caso de aneurisma de veia cava superior em 1950. Em 1968, May \& Nissel foram os primeiros a descrever o $\mathrm{AVP}^{8}$, e Dahl et al., os primeiros a relatar as complicações dos $\mathrm{AVP}^{8}$.

Não há um critério preciso quanto ao diâmetro do vaso para definir uma dilatação venosa como aneurismática. McDevitt propôs que tal definição seria correta diante de uma dilatação duas vezes maior do que o diâmetro normal ${ }^{9}$ do vaso. Sessa et al., em sua revisão, não concordaram com essa definição e citaram o critério de Maleti et al., que afirmam a necessidade desse diâmetro ser 3 vezes maior do que aquele da veia poplítea normal ${ }^{10}$ para tal consideração.

Diferentemente do sistema arterial, aneurismas do sistema venoso são raros e ocorrem em qualquer idade, sem distinção de sexo ${ }^{1}$. Não há concordância na literatura a respeito da localização mais freqüente dos aneurismas venosos. Perler et al. afirmam que são mais freqüentes nos membros superiores do que nos inferiores ${ }^{11}$; já Casttle \& Arous, em sua revisão, mostram que os aneurismas venosos são mais freqüentes nos membros inferiores $^{8}$.

Legnani ${ }^{12}$ relata que os AVP são os mais freqüentes nos membros inferiores, seguidos por aneurismas na veia safena magna e aneurismas venosos no pé. Em nosso meio, Dourado et al. descreveram dois casos de aneurismas venosos no nível do pé ${ }^{13}$. Os aneurismas venosos nos membros superiores geralmente estão associados a fístulas arteriovenosas para hemodiálise.

A maioria dos aneurismas venosos é, provavelmente, de origem congênita ${ }^{14}$, mas eles também podem 
ser resultado de alterações degenerativas ou de processos inflamatórios locais ${ }^{15}$, como trauma e infecção. Podem também estar associados a veias varicosas, devido a insuficiência valvular, processo obstrutivo ou então fístula arteriovenosa ${ }^{1}$.

A perda focal de componentes do tecido conjuntivo normal da parede da veia, devido a uma falha congênita de desenvolvimento ou perdas degenerativas associadas a idade, ou ainda a processo inflamatório, resulta no enfraquecimento da parede, tornando-a vulnerável à dilatação ${ }^{16}$.

Devido às alterações estruturais, os aneurismas venosos se apresentam de duas formas: fusiformes ou saculares. Essa diferenciação é importante para determinar a estratégia cirúrgica. Os AVP saculares representam $75 \%$ dos $\operatorname{casos}^{17}$.

Uma ampla variedade de apresentações clínicas dos aneurismas venosos é descrita na literatura ${ }^{11}$. A maioria dos casos de aneurismas venosos periféricos causa apenas desconforto local ${ }^{18}$, sendo, portanto, assintomática, com diagnóstico incidental ${ }^{11}$ (no caso apresentado, a paciente manifestou sintomas dolorosos no membro inferior esquerdo). No entanto, essa entidade nosológica pode manifestar-se por complicações, como trombose venosa profunda e embolia pulmonar, mais comumente.

Os aneurismas venosos periféricos podem ser detectados pela presença de massa subcutânea macia, que desaparece com a elevação do membro e aumenta com a manobra de Valsalva ${ }^{19}$. A suspeita será confirmada por ultra-sonografia vascular, atual método de esco1ha ${ }^{17}$ para o diagnóstico definitivo; a flebografia ascendente e a ressonância nuclear magnética ficam restritas aos casos de aneurismas venosos abdominais ou toráci$\cos ^{20,21}$. Entretanto, Coffman et al. ${ }^{22}$ consideram que a flebografia é mandatória para a determinação precisa da anatomia venosa antes da cirurgia.

Essa afecção deve ser lembrada no diagnóstico diferencial de massas nos membros inferiores, principalmente quando há alteração no tamanho conforme a posição do membro ${ }^{23}$.
Os achados histológicos revelam adelgaçamento na parede do vaso, endofleboesclerose e endoflebohipertrofia, um processo que poderia ser comparado com a aterosclerose $^{1,24}$. Revelam também hialinização da íntima associada a trombos, além de espessamento e fibrose da íntima, com diminuição acentuada das células musculares lisas da camada média, que pode estar delgada ou ausente em diferentes estágios, com presença ou não da limitante elástica interna ${ }^{25,26}$. A adventícia é, freqüentemente, fibrótica, com predomínio de fibras elásticas. As células inflamatórias, presentes principalmente nas áreas de trombose, também se encontram ao longo da parede da veia, na área aneurismática. Há predomínio de linfócitos, com poucos neutrófilos e raros eosinófilos. Mastócitos estão presentes na adventícia, aparentando não estarem aumentados em comparação com cortes de veias não-aneurismáticas ${ }^{27}$.

As complicações mais comuns dos aneurismas venosos são a trombose venosa profunda, a tromboflebite e a embolia pulmonar recorrente ${ }^{1}$. Alguns autores relatam que os aneurismas venosos grandes, assim como os saculares, apresentam maior predisposição para as complicações tromboembólicas ${ }^{28,29}$, embora não haja evidência clara sobre o diâmetro crítico ou sobre a forma do aneurisma que pode causar mais complicações. Chahlaoui et al.$^{30}$ relataram um caso fatal de paciente com múltiplos episódios de embolia pulmonar, portador de um aneurisma sacular pequeno $(20$ x $20 \mathrm{~mm})$; conclui-se daí que pequenos aneurismas também podem representar risco.

A cirurgia é o esteio do tratamento dos AVP. Em pacientes que apresentam complicações tromboembólicas, o tratamento cirúrgico é mandatório e considerado como de escolha, limitado apenas pelas condições clínicas $^{3-6,8,9,11-13,16,25,30,31-33}$, visto que a anticoagulação isolada nos pacientes com embolia pulmonar é ineficaz, com altos índices de recorrência $(80 \%)^{3,5,32,34}$. A indicação de tratamento cirúrgico em pacientes com AVP assintomático é contraditória. Entretanto, aneurismas saculares de qualquer tamanho e grandes aneurismas fusiformes deveriam ser tratados cirurgicamente, devido ao potencial de eventos tromboembólicos futu$\operatorname{ros}^{16,25}$. A recorrência de embolia pulmonar após cirurgia nunca foi descrita. Os AVP fusiformes pequenos e 
assintomáticos podem ser acompanhados clinicamente com estudos de eco-Doppler ${ }^{17}$ periódicos.

A aneurismectomia tangencial com venorrafia lateral é o método cirúrgico mais utilizado e tem sido recomendada para aneurismas saculares, mas também pode ser realizada em aneurismas fusiformes, como em nosso caso. A técnica foi descrita por Aldridge et al. ${ }^{27}$ e apresenta bons resultados.

A ressecção do aneurisma e a reconstrução venosa são recomendadas quando a aneurismectomia tangencial não pode ser realizada devido ao risco de permanência de segmento da doença na parede venosa. Esta seria uma conduta mais apropriada para a paciente em questão; porém, como não havia substituto autógeno para a reconstrução venosa, optou-se pela aneurismectomia tangencial. A reconstrução com prótese foi rejeitada devido ao risco aumentado de oclusão. $\mathrm{Na}$ casuística de $\mathrm{Sessa}^{25}$, três aneurismas fusiformes foram tratados com aneurismectomia tangencial. Várias opções cirúrgicas são passíveis de realização, tais como ressecção do aneurisma com anastomose terminoterminal, ressecção com interposição de enxerto venoso autógeno homólogo, ou então prótese de politetrafluoretileno (PTFE). São citadas também ligaduras com bypass venoso, ressecção com transposição venosa (tronco tibioperoneal para veia tibial anterior, veia do gastrocnêmio medial), ressecção sem reconstrução venosa e ligadura ${ }^{25}$. Deve ser citado ainda o implante de filtro de veia cava inferior ${ }^{25}$.

Embora a veia safena magna ipsilateral possa ser utilizada como substituta, essa estratégia deve ser evitada, pelo risco da perda de uma importante via colateral caso ocorra trombose após o reparo cirúrgico. A veia femoral superficial com válvula competente pode ser utilizada na ausência de veia safena ${ }^{33}$.

A perviedade da reconstrução da veia poplítea é pouco documentada na literatura. Os resultados da ressecção com anastomose terminoterminal não são conhecidos. O resultado no longo prazo parece ser satisfatório para a reconstrução com remendo venoso ou enxerto interposto de safena, diferente de quando se utilizam enxertos sintéticos e de veia jugular interna ${ }^{35}$.
O tratamento fibrinolítico já foi utilizado como parte inicial do tratamento em pacientes portadores de embolia pulmonar. Na literatura, poucos casos foram tratados com fibrinólise antes do reparo cirúrgico ${ }^{6,14,35}$. Essa terapia diminui ou elimina os trombos que eventualmente podem estar dentro do aneurisma, o que facilitaria o reparo cirúrgico ${ }^{26}$.

Também se deve citar o implante de filtro de veia cava inferior ${ }^{25}$, que pode ser uma alternativa para pacientes idosos, debilitados ou aqueles com contraindicação para anticoagulante, geralmente recomendada no pós-operatório.

\section{Conclusão}

AVP é uma ocorrência rara, que pode causar sérias complicações tromboembólicas. Essa afecção deve ser considerada em pacientes com episódios de embolia pulmonar de repetição e ausência de fatores de risco para tromboembolismo. O tratamento cirúrgico é indicado para todos os pacientes com AVP sintomático, devido ao risco elevado de recorrência de embolia pulmonar. Os AVP assintomáticos, porém grandes ou saculares, podem também ser tratados cirurgicamente. $\mathrm{O}$ tratamento mais utilizado é a aneurismectomia tangencial com venorrafia lateral. Entretanto, quando essa técnica não pode ser utilizada, deve-se fazer a ressecção com reconstrução. Os pequenos aneurismas, como os menores de $20 \mathrm{~mm}$ e sem trombos no seu interior, podem ser acompanhados com eco-Doppler seriado.

\section{Referências}

1. Ekim H, Gelen T, Karpuzoglu G. Multiple aneurysms of the cephalic vein. A case report. Angiology. 1995;46:265-7.

2. Dahl JR, Freed TA, Burke MF. Popliteal vein aneurysm with recurrent pulmonary thromboemboli. JAMA. 1976;236:2531-2.

3. Grice GD 3rd, Smith RB 3rd, Robinson PH, Rheudasil JM. Primary popliteal venous aneurysm with recurrent pulmonary emboli. J Vasc Surg. 1990;12:316-8.

4. Lang E, Bouwman O, Faiss J. [Recurrent lung embolisms in aneurysm of the popliteal vein]. Chirurg. 1993;64:503-4.

5. Donald IP, Edwards RC. Fatal outcome from popliteal venous aneurysm associated with pulmonary embolism. Br J Radiol. 1982;55:930-1.

6. Jack CR Jr., Sharma R, Vemuri RB. Popliteal venous aneurysm as a source of pulmonary emboli in a male: case report. Angiology. 1984;35:54-7. 
7. Sarap MD, Wheeler WE. Venous aneurysms. J Vasc Surg. 1988;8:182-3.

8. Castle JM, Arous EJ. Femoral vein aneurysm: a case report and review of the literature. Cardiovasc Surg. 1995;3:317-9.

9. McDevitt DT, Lohr JM, Martin KD, Welling RE, Sampson MG. Bilateral popliteal vein aneurysms. Ann Vasc Surg. 1993;7:282-6.

10. Maleti O, Lugli M, Collura M. Anévrysmes veineux popliteés: expérience personnelle. Phlebologie 1997;50:53-9.

11. Perler B. Venous aneurysm. An unusual upper-extremity mass. Arch Surg. 1990;125:124.

12. Legnani G, Mettini L, Bonfioli C, Motta F. Venous aneurysm of extremities: a case report and literature review. Cardiovasc Surg. 1995;3:445-6.

13. Dourado OC, Miranda AG, Pinheiro Filho A, Dourado LO. Aneurisma venoso no pé: relato de casos e revisão da literatura. J Vasc Bras 2006;5:313-6.

14. Dawson KJ, Hamilton G. Primary popliteal venous aneurysm with recurrent pulmonary emboli. J Vasc Surg. 1991;14:437.

15. Friedman SG, Krishnasastry KV, Doscher W, Deckoff SL. Primary venous aneurysms. Surgery. 1990;108:92-5.

16. Sessa C, Perrin M, Nicolini P. Anévrismes veineux. EMC Chirurgie. 2005;2:317-31.

17. Tambyraja AL, Moores C, Chalmers RT. Primary popliteal venous aneurysm. J Vasc Surg. 2004;40:830.

18. Gillespie DL, Villavicencio JL, Gallagher C, et al. Presentation and management of venous aneurysms. J Vasc Surg. 1997;26:845-52.

19. Calligaro KD, Ahmad S, Dandora R, et al. Venous aneurysms: surgical indications and review of the literature. Surgery. 1995;117:1-6.

20. Kassabian E, Coppin T, Combes M, Julia P, Fabiani JN. Radial nerve compression by a large cephalic vein aneurysm: case report. J Vasc Surg. 2003:38:617-9.

21. Schatz IJ, Fine G. Venous aneurysms. N Eng J Med. 1962;266:1310-2.

22. Coffman SW, Leon SM, Gupta SK. Popliteal venous aneurysms: report of an unusual presentation and literature review. Ann Vasc Surg. 2000;14:286-90.

23. Ranero-Juárez GA, Sánchez-Gómez RH, Loza-Jalil SE, Cano-Valdéz AM. Venous aneurysms of the extremities. Report of 4 cases and review of literature. Angiology. 2005;56:475-81.
24. Gruber HP, Amiri MA, Fraedrich G, Schlosser V. Multiple venous aneurysms of the saphenous vein: Report of an uncommon case. Vasc Surg. 1991;25:142-5.

25. Sessa C, Nicolini P, Perrin M, Farah I, Magne JL, Guidicelli H. Management of symptomatic and asymptomatic popliteal venous aneurysms: a retrospective analysis of 25 patients and review of the literature. J Vasc Surg. 2002;32:902-12.

26. Piccinato CE, Cherri J, Moriya T. Aneurisma venoso: relato de um caso. Rev Assoc Med Bras. 1986;32:71-2.

27. Aldridge SC, Comerota AJ, Katz ML, Wolk JH, Goldman BI, White JV. Popliteal venous aneurysm: report of two cases and review of the world literature. J Vasc Surg. 1993;18:708-15.

28. Yao JST, Van Bellen B, Flinn WR, Bergan JJ. Aneurysm of the venous system. In: Bergan JJ, Yao JST, eds. Aneurysms, diagnosis and treatment. New York: Grune \& Stratton; 1982. p. 515-29.

29. Leu AJ, Canova CR, Hoffmann U, Enzler M, Cassina PC. A soft popliteal mass and pulmonary embolism. Case report. Eur J Vasc Endovasc Surg. 1999;18:270-2.

30. Chahlaoui J, Julien M, Nadeau P, Bruneau L, Roy P, Sylvestre J. Popliteal venous aneurysm: a source of pulmonary embolism. AJR Am J Roentgenol. 1981;136:415-6.

31. Grice G, Smith R, Robinson P, et al: Primary popliteal venous aneurysm with recurrent pulmonary emboli. J Vasc Surg $1990 ; 12: 316-8$.

32. Greenwood LH, Yrizarry JM, Hallett JW Jr. Peripheral venous aneurysms with recurrent pulmonary embolism: report of a case and review of the literature. Cardiovasc Intervent Radiol. 1982;5:43-5.

33. Cheatle TR, Perrin M. Primary venous aneurysms of the popliteal fossa. Phlebology. 1993;8:82-5.

34. Banchini E, Villani LG, Rubini P. Anévrysmes de la veine poplitée. Description de 2 cas. J Mal Vasc. 1991;16:295-7.

35. Gallagher JJ, Hageman JH. Popliteal vein aneurysm causing pulmonary embolus. Arch Surg. 1985;120:1173-5.

Correspondência:

Fernando Thomazinho

Av. Voluntários da Pátria, 251

CEP 86061-160 - Londrina, PR

Tel.: (43) 3026.5254

Tel.: (43) 8402.6452

E-mail: thomazvase@hotmail.com 